\title{
Assessing the causal role of early visual areas in visual mental imagery
}

\author{
Paolo Bartolomeo (1), Dounia Hajhajate, Jianghao Liu and Alfredo Spagna
}

We read with great interest the review by J. Pearson on visual mental imagery (Pearson, J. The human imagination: the cognitive neuroscience of visual mental imagery. Nat. Rev. Neurosci. 20, 624-634 (2019)) $)^{1}$. The author outlines a model of visual mental imagery based on neuroimaging findings that involves large-scale brain networks spanning prefrontal areas to sensory areas, and that highlights the activation of occipital areas during visual mental imagery. Specifically, the model indicates that it is the "sensory and spatial representations of the imagery content" that would be formed in early visual areas.

However, individuals with acquired brain damage restricted to the occipital cortex typically have perfectly vivid visual mental imagery. For example, a patient with bilateral strokes in the white matter between the occipital and the temporal cortices ${ }^{2}$ had severe visual deficits for object form and colour, faces, words and letters but demonstrated perfectly preserved visual mental imagery abilities for these same items ${ }^{3}$. In addition, people with cortical blindness due to bilateral occipital lesions can experience vivid visual mental images ${ }^{4,5}$.

By contrast, patients with damage extending anteriorly in the temporal lobe, especially in the left hemisphere, often find themselves unable to build visual mental images ${ }^{6,7}$. Where does the discrepancy between the neuroimaging and neuropsychological findings come from? The neuroimaging results supporting the hypothesis of an implication of early visual areas are correlative in nature, whereas deficits in people with brain injury demonstrate a causal contribution of the lesioned circuits to the relevant cognitive ability (it is true that transcranial magnetic interference on the primary visual cortex was shown to impact visual mental imagery ${ }^{8}$, but this effect might depend on modulation of downstream visual areas).

A recent case report ${ }^{9}$ provided more specific evidence on the neural bases of visual mental imagery. After a bilateral stroke in the territory of the posterior cerebral artery, an architect, who before the stroke could easily imagine objects and buildings, spontaneously reported to have become unable to visualize items. By comparing his lesion location with those of other individuals with strokes in the same arterial territory, the authors found that the architect had selective damage in the left fusiform gyrus, a region in the ventral temporal cortex. The left temporal location is consistent with previous reports of individuals with impaired mental imagery after stroke $e^{6,7}$. During perception, this fusiform region might act as a neural interface between sensory information coming from the occipital cortex and semantic processing in the anterior temporal lobe ${ }^{10}$. In visual mental imagery, it could endow semantic memories with visual information. Taken together, the results from brain-damaged persons invite a revision of the neural model of visual mental imagery proposed by Pearson ${ }^{1}$, whereby fronto-parietal networks initiate, modulate and maintain activity in a core left temporal network centred on high-level visual regions in the ventral temporal cortex, with no causal role of early visual cortex.

There is a reply to this letter by Pearson J. Nat. Rev. Neurosci. https://doi.org/10.1038/ s41583-020-0349-4 (2020).
Paolo Bartolomeo (D) ${ }^{\text {凶}}$, Dounia Hajhajate ${ }^{1}$, Jianghao Liu ${ }^{1,2}$ and Alfredo Spagna ${ }^{1,3}$ Sorbonne Université, Inserm U 1127, CNRS UMR 7225, Paris Brain Institute, ICM, Hôpital de la Pitié-Salpêtrière, 75013, Paris, France.

${ }^{2}$ Dassault Systèmes, Vélizy-Villacoublay, France. ${ }^{3}$ Department of Psychology, Columbia University in the City of New York, New York, NY, USA 10027.

凶e-mail:paolo.bartolomeo@icm-institute.org https://doi.org/10 1038/s41583-020-0348-5

Pearson, J. The human imagination: the cognitive neuroscience of visual mental imagery. Nat. Rev. Neurosci. 20, 624-634 (2019).

2. Bartolomeo, P., Bachoud-Levi, A. C. \& Thiebaut de Schotten, M. The anatomy of cerebral achromatopsia: a reappraisal and comparison of two case reports. Cortex 56, 138-144 (2014)

3. Bartolomeo, P. et al. Multiple-domain dissociation between impaired visual perception and preserved mental imagery in a patient with bilateral extrastriate lesions. Neuropsychologia 36, 239-249 (1998).

4. Chatterjee, A. \& Southwood, M. H. Cortical blindness and visual imagery. Neurology 45, 2189-2195 (1995). de Gelder, B., Tamietto, M., Pegna, A. J. \& Van den Stock, J. Visual imagery influences brain responses to visual stimulation in bilateral cortical blindness. Cortex 72, 15-26 (2015)

6. Moro, V., Berlucchi, G., Lerch, J., Tomaiuolo, F. \& Aglioti, S. M. Selective deficit of mental visual imagery with intact primary visual cortex and visual perception. Cortex 44, 109-118 (2008).

7. Bartolomeo, P. The neural correlates of visual mental imagery: an ongoing debate. Cortex 44, 107-108 (2008).

8. Kosslyn S. M. et al. The role of area 17 in visual imagery: convergent evidence from PET and rTMS. Science 284, 167-170 (1999).

9. Thorudottir, S. et al. The architect who lost the ability to imagine: the cerebral basis of visual imagery. Brain Sciences 10, 59 (2020).

10. Ralph, M., Jefferies, E., Patterson, K. \& Rogers, T. T The neural and computational bases of semantic cognition. Nat. Rev. Neurosci. 18, 42 (2017).

Competing interests

The authors declare no competing interests

\section{Reply to: Assessing the causal role of early visual areas in visual mental imagery}

\section{Joel Pearson (D)}

In a recent review paper ${ }^{1}$, I outlined a model of visual mental imagery proposing a reverse visual hierarchy starting from prefrontal areas back to sensory areas.

I would like to thank Paolo Bartolomeo, Dounia Hajhajate, Jianghao Liu and Alfredo Spagna for their correspondence on our Review (The human imagination: the cognitive neuroscience of visual mental imagery. Nat. Rev. Neurosci. 20, 624-634 (2019)) $)^{1}$, which raises some important issues (Assessing the causal role of early visual areas in visual mental imagery. Nat. Rev. Neurosci. https:// doi.org/10.1038/10.1038/s41583-020-0348-5 $(2020))^{2}$.

Neuropsychological work reports that individuals with visual cortex damage can show some imagery-like processes without perception $^{3,4}$, while damage to the temporal lobe can correspond to a lack of imagery/ memory abilities ${ }^{5,6}$. Further, individuals with aphantasia (no imagery vividness or sensory imagery $)^{7,8}$ show normal sensory perception (although this is yet to be tested in detail), suggesting a general dissociation, and has been linked to areas outside of early visual cortex". Based on these reports Bartolomeo et al., suggest a revision to the reverse visual hierarchy model I described, without a causal role of early visual cortex. Here I outline three reasons why this is not required.

Much of the neuropsychological work on imagery is actually correlational, often documented brain injury is correlated with 
subjective descriptions of imagery loss. Hence, we do not know an individual's imagery abilities prior to the injury. For it to be causal, visual imagery would need to be accurately assessed before and after predefined or 'planned' and controlled damage or interruption to a particular brain region (or with a control site), in a similar manner to TMS work in humans or animal lesion work.

Perhaps more of a problem for imagery research is the variety of different ways it is measured $^{1}$. Most typically in neuropsychological work discussed by Bartolomeo et al., subjective reports are used, perhaps most problematic are questions regarding the physical structure of objects as $\mathrm{in}^{4,10}$, which use questions like "Do tractors have two large wheels at the back or front?" Such knowledge questions can easily be answered without imagery. In contrast to this, much of the behavioural and fMRI work use objective task-based measures $^{11}$. This is important because research suggests that these different measures do not rely on the same brain areas ${ }^{1,12}$. This measurement problem is well illustrated by the fact that many aphantasics always believed they had mental imagery, not fully understanding the sensory nature of imagery in others until it was graphically described.

Interestingly, new data indeed suggest a causative link between cortical excitability in early visual cortex and measures of imagery strength. Recent work has shown that the excitability of early visual cortex predicts and can modulate imagery strength in a 'causal' manner via brain stimulation ${ }^{13}$.

One way to reconcile these different findings is that early visual cortex has a causative role in the high definition precise features of visual imagery, but not lower fidelity imagery and not necessarily reports of imagery vividness or object descriptions. With such a model damage to primary visual cortex would result in a loss of the high-fidelity precise dimensions of imagery, if an individual did indeed have this to begin with, but not a loss in high-level imagery of places, faces, spatial imagery or imagery vividness. To put this another way, visual imagery, like visual perception, is not a unitary process. Different features, colour, form and motion, and their levels of precision are processed across different brain areas and most likely use a range of mechanisms. Further complicating this hierarchical spread is the extreme range of individual differences that naturally exist with imagery, together making cross-methodological comparisons and meta-analyses difficult.

$$
\begin{array}{r}
\text { Joel Pearson iD } \\
\text { School of psychology, The University of New South } \\
\text { Wales, Sydney, Australia. } \\
\text { e-mail: jpearson@unsw.edu.au }
\end{array}
$$

1. Pearson, J. The human imagination: the cognitive neuroscience of visual mental imagery. Nat. Rev. Neurosci. 20, 624-634 (2019).

2. Bartolomeo, P., Hajhajate, D., Liu, J. \& Spagna, A. Assessing the causal role of early visual areas in visual mental imagery. Nat. Rev. Neurosci. https://doi.org/ 10.1038/s41583-020-0348-5 (2020).

3. de Gelder, B., Tamietto, M., Pegna, A. J. \& Van den Stock, J. Visual imagery influences brain responses to visual stimulation in bilateral cortical blindness. Cortex 72, 15-26 (2015).

4. Chatterjee, A. \& Southwood, M. H. Cortical blindness and visual imagery. Neurology 45, 2189-2195 (1995).

5. Moro, V., Berlucchi, G., Lerch, J., Tomaiuolo, F. \& Aglioti, S. M. Selective deficit of mental visual imagery with intact primary visual cortex and visual perception. Cortex 44, 109-118 (2008).

6. Bartolomeo, P. The neural correlates of visual mental imagery: An ongoing debate. Cortex 44, 107-108 (2008).

7. Zeman, A., Dewar, M. \& della Sala, S. Lives without imagery - congenital aphantasia. Cortex 73. 378-380 (2015)

8. Keogh, R. \& Pearson, J. The blind mind: no sensory visual imagery in aphantasia. Cortex 105, 53-60 (2017).

9. Thorudottir, S. et al. The architect who lost the ability to imagine: the cerebral basis of visual imagery. Brain Sciences 10, 59-15 (2020).

10. Bartolomeo, P. et al. Multiple-domain dissociation between impaired visual perception and preserved mental imagery in a patient with bilateral extrastriate lesions. Neuropsychologia 36, 239-249 (1998).

11. Pearson, J. New directions in mental-imagery research: the binocular-rivalry technique and decoding fMRI patterns. Curr. Dir. Psychol Sci. 23, 178-183 (2014).

12. Bergmann, J., Genc, E., Kohler, A., Singer, W. \& Pearson, J. Smaller primary visual cortex is associated with stronger, but less precise mental imagery. Cerebral Cortex 26, 3838-3850 (2016).

13. Keogh, R., Bergmann, J. \& Pearson, J. Cortical excitability controls the strength of mental imagery. elife 9, e50232 (2020).

Competing interests

The author declares no competing interests. 